\title{
A Phased Approach to Unlocking During the Covid-19 pandemic - Lessons from Trend Analysis
}

\author{
Mike Stedman ${ }^{1}$, Mark Davies ${ }^{1}$, Mark Lunt ${ }^{2}$, Arpana Verma ${ }^{2}$, Simon Andersen $^{3}$, and \\ Adrian Heald ${ }^{4}$ \\ ${ }^{1}$ Res Consortium \\ ${ }^{2}$ The University of Manchester \\ ${ }^{3}$ University of West Indes \\ ${ }^{4}$ Salford Royal Hospitals NHS Trust
}

April 28, 2020

\begin{abstract}
Background The COVID-19 pandemic has led to radical political control of social behaviour. The purpose of this paper is to explore data trends from the pandemic regarding infection rates/policy impact, and draw learning points for informing the unlocking process. Methods The daily published cases in England in each of 149 Upper Tier Local Authority (UTLA) areas were converted to Average Daily Infection Rate(ADIR), an R-value - the number of further people infected by one infected person during their infectious phase with Rate of Change of Infection Rate(RCIR) also calculated. Stepwise regression was carried out to see what local factors could be linked to differences in local infection rates. Results By the 19th April 2020 the infection $\mathrm{R}$ has fallen from 2.8 on 23rd March before the lockdown and has stabilised at about 0.8 sufficient for suppression. However there remain significant variations between England regions. Regression analysis across UTLAs found that the only factor relating to reduction in ADIR was the historic number of confirmed number infection/000 population, There is however wide variation between Upper Tier Local Authorities (UTLA) areas. Extrapolation of these results showed that unreported community infection may be $>200$ times higher than reported cases, providing evidence that by the end of the second week in April, $29 \%$ of the population may already have had the disease and so have increased immunity. Conclusion Analysis of current case data using infectious ratio has provided novel insight into the current national state and can be used to make better-informed decisions about future management of restricted social behaviour and movement.
\end{abstract}

\section{A phased approach to unlocking during the COVID-19 pandemic - Lessons from trend analysis}

Mike Stedman ${ }^{1}$, Mark Davies ${ }^{1}$, Mark Lunt ${ }^{2}$, Arpana Verma ${ }^{3}$, Simon G. Anderson ${ }^{4,5}$, Adrian H Heald ${ }^{6,7}$

${ }^{1}$ Res Consortium, Andover, Hampshire; ${ }^{2}$ University of Manchester, Division of Musculoskeletal and Dermatological Sciences; ${ }^{3}$ Population Health, Health Services Research and Primary Care, The University of Manchester, Manchester, United Kingdom ${ }^{4}$ University of the West Indies, Cave Hill Campus Barbados; 5Division of Cardiovascular Sciences, Faculty of Biology, Medicine and Health, The University of Manchester, Manchester, United Kingdom; ${ }^{6}$ Department of Diabetes and Endocrinology, Salford; ${ }^{7}$ The Faculty of Biology, Medicine and Health and Manchester Academic Health Sciences Centre, University of Manchester, UK

Key Words: COVID-19, Unlock, Epidemiology, Strategy

Word Count: 3010

Number of figures: 4 
Corresponding Author: Dr Adrian Heald, Department of Diabetes and Endocrinology, Salford Royal Hospital, Salford UK M6 HD

Telephone: +441612065157

Email: adrian.heald@manchester.ac.uk

\section{Abstract}

\section{Background}

The COVID-19 pandemic has led to radical political control of social behaviour. The purpose of this paper is to explore data trends from the pandemic regarding infection rates/policy impact, and draw learning points for informing the unlocking process.

\section{Methods}

The daily published cases in England in each of 149 Upper Tier Local Authority (UTLA) areas were converted to Average Daily Infection Rate(ADIR), an R-value - the number of further people infected by one infected person during their infectious phase with Rate of Change of Infection Rate(RCIR) also calculated.

Stepwise regression was carried out to see what local factors could be linked to differences in local infection rates.

\section{Findings}

By the $19^{\text {th }}$ April 2020 the infection $\mathrm{R}$ has fallen over the from 2.8 on $23^{\text {rd }}$ March before the lockdown and has stabilised at about 0.8 sufficient for suppression. However there remain significant variations between England regions.

Regression analysis across UTLAs found that the only factor relating to reduction in ADIR was the historic number of confirmed number infection/000 population, There is however wide variation between Upper Tier Local Authorities (UTLA) areas. Extrapolation of these results showed that unreported community infection may be $>200$ times higher than reported cases, providing evidence that by the end of the second week in April, $29 \%$ of the population may already have had the disease and so have increased immunity.

\section{Interpretation}

Analysis of current case data using infectious ratio has provided novel insight into the current national state and can be used to make better-informed decisions about future management of restricted social behaviour and movement.

\section{Funding}

There was no external funding for this work

\section{Research in Context}

\section{Evidence before this study}

- Following the first recorded cases of SARS-CoV-2 on the $29^{\text {th }}$ January in the United Kingdom (UK), the COVID-19 pandemic has taken a rapidly developing course culminating in a comprehensive populationwide lockdown on the $23^{\text {rd }}$ March, 2020.

- The toll on health and lives has been significant in the UK and elsewhere in the world.

- The ongoing rate of infection is determined to a large extent by the R-value of an infectious disease. The R-value is the number of people infected by one infected person during their infectious phase.

\section{Added value of this study}

- The peak of COVID-19 infection has passed. 
- The infection rate $\mathrm{R}$ has fallen from before shutdown $23^{\text {rd }}$ March 2020 value of 2.8 to now a suppression value of 0.8 .

- Our modelling indicates that only one factor, the total reported cases /,000 population is significantly associated with the daily infection rate.

- We predict that there are as many as 237 community cases for each reported case. With the current 73,000 reported cases this suggests that $29 \%$ of the UK population has already been infected.

\section{Implications}

- We believe that several key principles can be derived from the analysis which may aid policy makers in a smoother transition to reducing social containment and sustainably managing the COVID-19 disease.

- These principles include focusing on achieving low enough $\mathrm{R}$ values to keep mortality comparable with influenza, tailoring social behavioural policies to the ongoing tally of latest case numbers and calculating the current R-value within each locality.

\section{Background}

Severe Acute Respiratory Syndrome Coronavirus-2 (SARS-CoV-2) is the name given to the 2019 novel coronavirus. COVID-19 is the name given to the disease associated with the virus. The COVID-19 pandemic has led to radical political control of social behaviour across the world. SARS-CoV-2 is a new strain of coronavirus that has not been previously identified in humans. Following the first recorded cases of SARSCoV-2 on the $29^{\text {th }}$ January in the United Kingdom, the COVID-19 pandemic has taken a rapidly developing course with a switch by the United Kingdom (UK) Government on the $17^{\text {th }}$ March from a policy of "track and containment" to "mitigation" and initiated social distancing, followed by a comprehensive population lockdown on the $23^{\text {rd }}$ March. The toll on health and lives has been very significant in the UK and elsewhere in the world (1). High-risk groups, based on age and underlying comorbidities, were told to isolate themselves completely for the next 13 weeks $(2,3)$. The rationale was to reduce the impact of the high growth phase of the pandemic on the National Health Service (NHS) with particular focus on Intensive Care Units (ICUs) and High Dependency Units (HDUs) and to keep mortality to a minimum $(4,5,6)$.

Pandemic models forecast that with continuing progress the social lockdown would be relaxed when there is clear evidence of a downturn in infection rates and mortality. There is a trade-off here between balancing the clinical impact of the pandemic with the economic, social and longer-term healthcare impact. This includes considering the impact on diverting resources away from mainstream severe and long-term conditions within primary and secondary care, as well as recognising that the capacity of the population to maintain confinement is limited.

Testing in the very initial phases was carried out on the wider groups who had contacts with diagnosed patients. Testing capacity initiatives have been slow to appear with testing at 5,000/day at the end of March increasing to 10,000/day in April (7). As number of new cases grew and testing capacity limitation was reached testing was restricted to symptomatic hospital-based patients, and more recently as numbers have fallen and testing capacity increased to general practice presentations and NHS staff. Using the total confirmed cases as a sample of the overall levels of population infection is reasonable if the selection rules are consistently applied both over time and geography. While there may be some variations, selection for testing was being restricted during the growth phase and then increased as numbers fall. This will have first reduced and now increase the numbers of new cases identified. The direction of any error would therefore be to initially to show lower and now relatively higher infection rates.

Given the past community based 3-day doubling infection rate, there are indications that significant part of the population may already have been infected with low grade clinical or subclinical symptoms. This wider non-hospitalised population is likely to continue to grow even with the isolation and social distancing policies.

The ongoing rate of infection is determined to a large extent by the R-value of an infectious disease. The $\mathrm{R}$-value is the number of people infected by one infected person during their infectious phase (8). This value 
is dependent on the level of local and cross-community social contacts and the proportion of the current population who have not developed immunity through previous exposure. An R-value above 2 suggests more than a doubling of people with the condition during each infectious period and an R-value below 1 is consistent with "suppression" meaning that the virus prevalence will slowly diminish.

The purpose of this paper is to briefly explore data trends from the pandemic in terms of infection rates and policy impact and draw learning points for informing the unlocking process.

\section{Methods}

In England, local government is divided between an Upper Tier (county council) and a Lower Tier (district council). The data of COVID-19 case are published daily for each of the 149 Upper Tier Local Authorities (UTLA) (9) which vary in size from $1.6 \mathrm{~m}$ to $97 \mathrm{k}$. This study used the latest data download possible.

The UTLA population numbers were taken from GP practice patient numbers published by Lower Layer Super Output Area (LSOA) then aggregated up to their respective UTLA $(10,11)$. The same method was used to aggregate the other population demographic and health characteristics to UTLA level.

Statistical Analysis

The new cases were calculated and plotted on a timeline with a simple polynomial trend analysis. An exponential curve based on the disease 3 days doubling characteristic linked to the starting data was included for reference.

Two further variables the Average Daily Infection Rate (ADIR) and the Rate of Change of Infection Rate ([?]IR) were calculated and used to track the national and regional developments in infection rate.

The COVID-19 characteristics incorporated into the analysis are 5 Incubation days and 5 Infectious days. Similar assumptions were made by the Imperial College COVID-19 Response Team in their 'Impact of nonpharmaceutical interventions (NPIs) to reduce COVID-19 mortality and healthcare demand' (12). In that paper, the authors stated "We assumed an incubation period of 5.1 days. Infectiousness is assumed to occur from 12 hours before the onset of symptoms for those that are symptomatic and from 4.6 days after infection in those that are asymptomatic with an infectiousness profile over time that results in a 6.5 -day mean generation time".

Average Daily Infection Rate $\left(\mathrm{R}_{\mathrm{ADIR}}\right)$ : The daily infection rate $\mathrm{R}$ on any given day is calculated by dividing the infected population i.e. the reported new cases 5 days ahead (corresponding to the incubation period), by the infectious population i.e. an average of new cases over the 5 previous days (corresponding to the infectious period). $R_{\mathrm{ADIR}}$ is taken as a rolling average of the $\mathrm{R}$ values over the previous 7 -days to allow for variation in weekly administrative case count. Therefore

- $\mathrm{n}=$ Date of Infection

- Total Cases $(\mathrm{TC})=$ Daily Reported Total Cumulative Confirmed Cases

- New Cases $(\mathrm{NC})=\mathrm{TC}_{(\mathrm{n})}-\mathrm{TC}_{(\mathrm{n}-1)}$

- Average Infectious group (AI) $=\sum_{n-5}^{n} \mathrm{NC} / 5$ Infectious days

- Infection Ratio $(\mathbf{R})=\mathbf{N C}\left(\mathbf{n}+5\right.$ Incubation Days) $/ \mathbf{A I}_{(\mathbf{n})}$

- $\mathbf{R}_{\mathrm{ADIR}(\mathrm{n})}=\sum_{\mathbf{n}-6}^{\mathrm{n}} \mathbf{R} / \mathbf{7}$ days in week

Two sensitivity analyses were considered, first where the condition is faster in incubation and infection (4 days for each) and second where the condition is slower (6 days for each).

Rate of Change of Infection Rate $(\Delta \mathrm{IR})$ : is calculated by taking the slope of the least-squares fit line using the previous 7 days $R$ values.

The relation between the $\mathrm{R}_{\mathrm{ADIR}}$ and the $\Delta \mathrm{IR}$ in all the UTLAs was determined and the impact of the disease progression was considered by including the total number of reported cases/,000 population, shown in quartiles. 
A stepwise regression model linking the $\mathrm{R}_{\mathrm{ADIR}}$ to the local community characteristics with weighting by population numbers was carried out with factors included

- Location: Urban/Rural, Latitude/Longitude, occupants/Household taken from the Office of National Statistics

- Demographics: \% Individual with Age $>60$, Social Deprivation, \% in full-time employment or education, Ethnicity, were taken from NHS GP Practice profiles

- Health: \% population with Longer term conditions (including hypertension and diabetes), $\%$ confident in their own health management were taken from GP Practice Profiles

- COVID-19: Total Reported cases/1,000 population taken from this study 8 April 2020

Local GP practice data taken from various sources were aggregated up to UTLA level.

The regression coefficients for the association between $\mathrm{R}_{\mathrm{ADIR}}$ and the reported COVID-19 cases/population were used to determine the $\mathrm{R}_{\mathrm{ADIR}}$ when an UTLA has no reported cases. This is the expected value that the lockdown and increased social distancing delivers on their own on this day. One can also extrapolate to a value of Cases/1,000 pop that would be needed to give a $R_{\text {ADIR }}=0$ i.e. $100 \%$ immunity in the total population. This value can then be used to indicate the relation between reported and community infection levels. Linear extrapolation was used however there may be asymptotic effects that change this number.

The Office of National Statistics has reported a detailed analysis of the total mortality in March associated with COVID-19 (13). This total additional mortality can be related to the total end of March reported cases of COVID-19 which can be uprated by the total potential community infection rate calculated in this report to give an estimate of overall COVID mortality rate.

Patients or the public WERE NOT involved in the design, or conduct, or reporting, or dissemination plans of our research. Ethics permission was not required, as no individually identifiable data were included in the analysis nor were nay individuals contacted.

\section{Results}

As of the $24^{\text {th }}$ April 2020, the number of a confirmed case of COVID-19 in England stands at 104,565 with 16,996 deaths. These covered a total recorded population of 60 million.

Figure 1 shows the reported new cases of COVID-19 have peaked on the $8^{\text {th }}$ April and with reported deaths from COVID-19 which lags new cases by a week have also peaked.

Analysis for $19^{\text {th }}$ April 2020 (5 days before the latest new cases presentation) shows the national $\mathrm{R}_{\mathrm{ADIR}}$ was 0.81 with $\Delta \mathrm{IR}$ at -0.03 . In figure 2 , the $\mathrm{R}_{\mathrm{ADIR}}$ is plotted against time, highlighting that the daily $\mathrm{R}$-value breakthrough below 1 was also achieved on the $8^{\text {th }}$ April 2020.

The results of this analysis as shown in Figure 2 show a steady level $R_{\text {ADIR }}$ below 3 before the 24/3/2020 lockdown and then a steady fall $\mathrm{R}_{\mathrm{ADIR}}$ after that. The current infection $\mathrm{R}_{\mathrm{ADIR}}$ has stabilised at around 0.8. However, given the strong likelihood that the virus will become endemic, a reproductive rate of 0 is unrealistic. Nevertheless, keeping the ongoing $R_{\mathrm{ADIR}}$ value below 1 is an opportunity to reduce rapid re-emergence adopted by the UK Government.

Figure 3 shows the differences in UTLAs of $R_{\text {ADIR }}$ and $\Delta I R$. This shows that there is a wide difference between UTLAs with over 30 of them still with infection rates above 1 but decreasing, while other regions already well below 1 and decreasing more slowly. There remain some regions where progress is slower. The inclusion of the number of reported cases/1,000 population quartiles show that those regions with the highest cases $/ 1,000$ population now have the lowest infection rates, suggesting there may be a relationship between these two factors.

The stepwise regression of the local UTLA factors to $\mathrm{R}_{\mathrm{ADIR}}$ showed that only one factor total reported cases/1,000 population was significantly linked. In Figure 4, the regression, weighted by local UTLA population, had an $\mathrm{r}^{2}=0.22$, $\mathrm{p}$ value $<0.0001$ and the standardised beta of -0.42 . Of note here is that the analysis is 
carried out 5 days before the latest data as, due to the incubation period, that is when the relevant infections would have taken place, and the latest data itself is also subject to ongoing updates.

The regression results in an equation $\mathrm{R}_{\mathrm{ADIR}}=1.06-0.16 \times$ Current Total Cases/1,000 population. The reported cases are an unknown fraction of the total community cases. However, one can see that without any reported cases (i.e. no reduced community immunity) a UTLA would have $R_{\text {ADIR }}$ of 1.0 - thus the implementation of social distancing has delivered a substantial reduction from the historic $\mathrm{R}$ with low number of existing cases at 2.8 (figure 2).

If this relationship is linear then extrapolation (See Figure 4) shows zero $R_{\mathrm{ADIR}}$ being achieved at 6.6 reported cases /1,000 community population. Therefore, to achieve full population immunity, this is equivalent to 150 community cases for each reported case. Total reporting of 400,000 confirmed cases would be expected if the total population of 60 million achieved increased immunity. This also suggests with current 105,000 reported cases that 16.1 million ( $26.8 \%$ of the total population) have now been infected.

Applying the 150 difference between community infection and reported cases can also be used to examine mortality. The ONS reported between 1 and 31 March 2020, there was a total of 47,358 deaths. Of these, 3,912 deaths (8\%) were reported to have involved the coronavirus (COVID-19)). There were a total 12,288 reported cases of COVID-19 up to 5 days before the end of March; this, according to the above factor (150), is equivalent to a community infection of around 2.0 million people. This reflects a mortality rate of $0.2 \%$ in the total infected population. If this rate is applied to the total 60 million population then up to 120,000 are at risk of dying.

\section{Discussion}

Having a clear understanding of the historic recovery in the community is a critical piece of information to policymakers as higher levels mitigate the impact associated with relaxing the social constraints. A published piece of work not yet reviewed shows serology results from 1/4/2020 carried out on 3,300 people in Santa Clara California that show 40-80 times as many people in the community have had the disease than was reported by their testing program (14)

The analysis shown in Figure 2 highlights that current lockdown measures are reducing the daily R-value down to well below one. However, to commence relaxing these measures, we suggest several principles need to be in place to ensure the R-value of COVID-19 does not rise above 1, triggering a second pandemic (there is general acceptance that the disease will inevitably become endemic).

Figure 3 highlights how the disease progression varies across UTLAs and how that impacts the infection rate and its relative speed of change. Regions with history of the most cases/population have the lowest infection rate $R_{\text {ADIR }}$ and lowest rate of change in infection rate $\Delta I R$.

Social distancing behaviour and rules implementation could be expected to vary across different communities/groups, and as the different UTLAs have varying amounts of these different communities, examining the variation of infection rate across UTLAs one would hope to see which community groups were responding well and which were responding less well to social distancing. Figure 4 shows the only factor that could be related to the $\mathrm{R}_{\mathrm{ADIR}}$ in this analysis was the historic number of confirmed number infection/,000 population suggesting that some of the reduction in reported cases is due to the build-up of immunity due to larger numbers of historic cases in the population.

An important comparative R-value reference would be another coronavirus endemic infection, influenza. During seasonal periods, research indicates that influenza has an R-value of around 1.3 (15) and can result in the highest periods up to 200 additional deaths per day above mortality from other causes, although these figures are constrained by the provision of flu vaccine which is available particularly for the high-risk group. However, if the current pandemic can be switched to a similar mortality rate (with carefully phases social behaviour policies in place, along with population testing) then unlocking can be managed in a politically and socially acceptable way. Some observations around this included 
1. The principle of self-isolation following infection/symptoms is now well in place in the population

2. The track and contain mechanisms to identify next line contacts of infected people can also be increased with technical support

3. The vulnerable groups can continue to be isolated with their 13-week restriction kept in place but supported by the general population

4. Health service is now better able to cope with the load

Adding to this, experiences with different pandemic policy frameworks suggest that a looser more flexible approach to social activity can be managed if high-risk groups are more carefully protected. This is particularly pertinent given the news this week that elderly care homes are a significant area of both infections and pandemic mortality (16).

The speed of the unlocking process will depend on the level of unlocking. However, what is clear is that with the potentially reduced at-risk population any further peaks will be lower. We looked in UTLAs at the potential determinants of the ADIR and found that the only factor that related to this was the historic number of confirmed number infection/,000 population. This suggests that removing the lockdown from areas with higher historic caseloads should present a lower risk of R-value reversal.

However, a 'one size fits all' approach to pandemic policy does not consider the variation in both infection rates and impact across localities. When the data at the regional level is analysed there seems to be a wide variety of $\mathrm{R}$-values and slope of extrapolated $\mathrm{R}$-line over time, implying that unlocking needs to have a certain level of 'tailoring' of social behavioural policies and testing to be effective. These differences are likely to be due to differences in local factors such as infection drivers and underlying population morbidities. This has been explored in a separate publication by the same authors (17).

\section{Strengths}

The strength of this paper is that we have utilised the actual available national level England data pertaining to COVID-19 reported and infected cases and deaths in relation to quantifiable population factors. Also we have factored demographic and health factors into the analysis.

\section{Limitations}

A weakness is the assumption that the limited number of new confirmed cases is consistent related to the infected numbers in the general population both over time and geography - in other words that the relationship shown between number of reported cases / 1,000 population and infection ratio can be extrapolated in linear fashion. Furthermore we have not made any comparison with other parts of Europe where lockdown and testing strategies have differed from the UK.

\section{Conclusion}

Unlocking current social restrictions as soon as possible is vital to minimise demand on the economy and the impact of prolonged social containment. However, this must be balanced against containing the current pandemic and minimising future infection waves.

While mindful of the limitations of trend analysis, we believe that several key principles can be derived from the analysis which may aid policy makers in a smoother transition to reducing social containment and sustainably managing the COVID-19 disease. These principles include focusing on achieving low enough $\mathrm{R}$ values to keep mortality comparable with influenza, tailoring social behavioural policies to the ongoing tally of latest case numbers and calculating the current R-value within each locality.

We hope this analysis will have relevance and utility for policymakers at national and regional levels in managing the population 'Unlock' across the UK and elsewhere.

\section{Figure Legends}

Figure 1 Daily Confirmed New Cases, Model of exponential Case Growth and Confirmed Deaths over the last 6 weeks in England 
Figure 2 Average Daily Infection Rate $\left(\mathrm{R}_{\mathrm{ADIR}}\right)$ for the base model and 2 sensitivity cases

Figure 3: The latest Average Daily Infection Rate $\left(\mathrm{R}_{\mathrm{ADIR}}\right)$ versus the Rate of Change of Infection Rate $(\triangle \mathrm{IR})$ for each UTLA divided into quartiles for total reported cases/1,000 population

Figure 4: Average Daily Infection Rate $\left(\mathrm{R}_{\mathrm{ADIR}}\right)$ for Upper Tier Local Authority linked to Total reported cases $/ 1,000$ population

Supplementary Table: List of Total Cases and deaths by day and calculated $\mathrm{R}_{\mathrm{ADIR}}$ and $\Delta \mathrm{IR}$

\section{Funding}

No external funding was used in relation to the funding of the work leading to this paper.

\section{Author's Contributions}

Mike Stedman drove this paper and carried out the main analysis and wrote the paper. Mark Davies co-wrote the paper and designed the discussion. Mark Lunt provided statistical overview and editorial input. Arpana Verma reviewed the whole manuscript and provided contextual senior review. Simon Anderson reviewed the manuscript and also supervised the statistical analysis. Adrian Heald co-wrote the manuscript and provided clinical context and data interpretation.

\section{Disclaimers}

Neither Patients nor the public was involved in the design, or conduct, or reporting, or dissemination of our findings.

The manuscript is an honest, accurate, and transparent account of the study being reported. No important aspects of the study have been omitted.

\section{References}

1. World Health Organization, Coronavirus disease (COVID-2019) situation reports; https://www.who.int/emergencies/di coronavirus-2019/situation-reports/ [accessed 15 April 2020].

2. Mahase E. COVID-19: the UK starts social distancing after new model points to 260000 potential deaths. BMJ2020;368:m1089. doi:10.1136/bmj.m1089 pmid:32184205

3. Kmietowicz Zosia. COVID-19: Highest risk patients are asked to stay at home for 12 weeks BMJ 2020; 368: $\mathrm{m} 1170$

4. C. Fraser, S. Riley, R. M. Anderson, N. M. Ferguson, Factors that make an infectious disease outbreak controllable. Proc. Natl. Acad. Sci. U.S.A. 101, 6146-6151 (2004). doi:10.1073/pnas.0307506101pmid:15071187

5. Anderson RM, Fraser F et al. Epidemiology, transmission dynamics and control of SARS: the 20022003 epidemic. Phil Trans Roy Soc Ser B 2004; 359: 1091-490.

6. Public Health England. COVID-19: guidance on shielding and protecting people defined on medical grounds as extremely vulnerable. 30 Mar 2020.

7. https://www.gov.uk/government/publications/guidance-on-shielding-and-protecting-extremely-vulnerablepersons-from-covid-19

8. https://assets.publishing.service.gov.uk/government/uploads/system/uploads/attachment_data/file/878121/coronavir covid-19-testing-strategy.pdf

9. Infect Dis Model. 2017 Aug; 2(3): 288-303. Published online 2017 Jun 29. doi: 10.1016/j.idm.2017.06.002

PMCID: PMC6002118 PMID: 29928743 Reproduction numbers of infectious disease models Pauline van den Driessche

10. COVI19 case \& death data https://coronavirus.data.gov.uk/

11. Population number in GP practice Lower Layer Super Output Areas (LSOA) in April 2020 https://digital.nhs.uk/dataand-information/publications/statistical/patients-registered-at-a-gp-practice/april-2020

12. LSOAs link to individual UTLA https://geoportal.statistics.gov.uk/datasets/lower-layer-super-outputarea-2011-to-upper-tier-local-authorities-2019-lookup-in-england-and-wales-/data 
13. https://www.imperial.ac.uk/media/imperial-college/medicine/sph/ide/gida-fellowships/Imperial-CollegeCOVID19-NPI-modelling-16-03-2020.pdf

14. https://www.ons.gov.uk/peoplepopulationandcommunity/birthsdeathsandmarriages/deaths/bulletins/deathsinvolving

15. Bhattacharya et al COVID-19 Antibody Seroprevalence in Santa Clara County, California Dept of Medicine Stanford University doi.org/10.1101/2020.04.14.20062463.

16. https://www.ncbi.nlm.nih.gov/pmc/articles/PMC4169819/)

17. UK care homes struggle with wave of COVID-19 cases Financial Times 14 April https://www.ft.com/content/574ca84ae735-4e42-8faf-62c641953efc

18. M Stedman, M Lunt, M Davies, M Gibson, A Heald COVID-19: Modelling Local Transmission and Morbidity effects to provide an estimate of overall Relative Healthcare Resource Impact by General Practice Granularity doi: https://doi.org/10.1101/2020.03.20.20039024
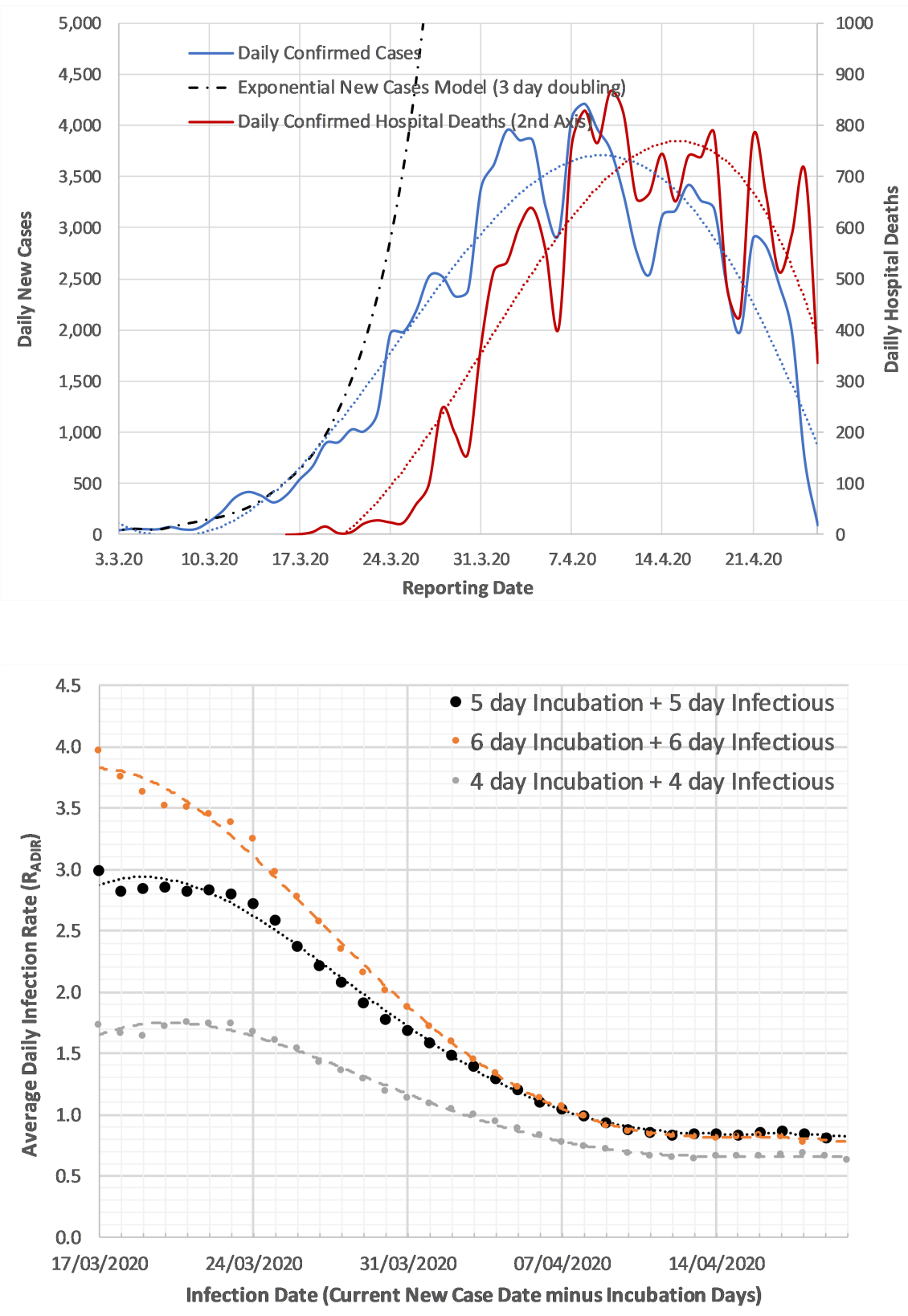

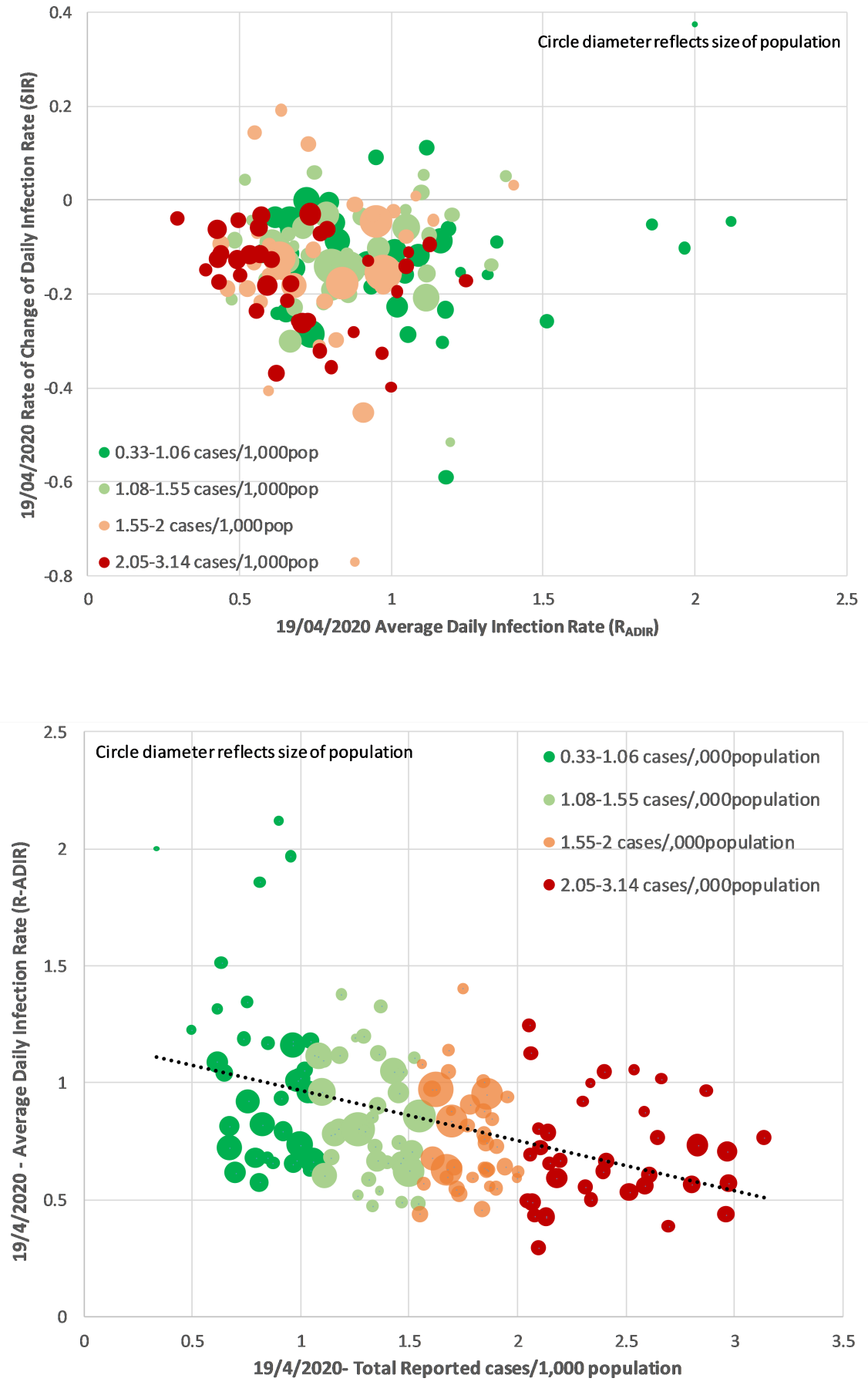